M.M. Nachit • I. Elouafi • M.A. Pagnotta $\cdot$ A. El Saleh

E. Iacono $\cdot$ M. Labhilili $\cdot$ A. Asbati $\cdot$ M. Azrak

H. Hazzam • D. Benscher • M. Khairallah

J.-M. Ribaut $\cdot$ O.A. Tanzarella $\cdot$ E. Porceddu

M.E. Sorrells

\title{
Molecular linkage map for an intraspecific recombinant inbred population of durum wheat (Triticum turgidum L. var. durum)
}

Received: 14 February 2000 / Accepted: 28 April 2000

\begin{abstract}
Durum wheat (Triticum turgidum L. var. durum) is an economically and nutritionally important cereal crop in the Mediterranean region. To further our understanding of durum genome organization we constructed a durum linkage map using restriction fragment length polymorphisms (RFLPs), simple sequence repeats (SSRs) known as Gatersleben wheat microsatellites (GWMs), amplified fragment length polymorphisms (AFLPs), and seed storage proteins (SSPs: gliadins and glutenins). A population of $110 \mathrm{~F}_{9}$ recombinant inbred lines (RILs) was derived from an intraspecific cross between two durum cultivars, Jennah Khetifa and Cham 1. The two parents exhibit contrasting traits for resistance to biotic and abiotic stresses and for grain quality. In total, 306 markers have been placed on the linkage map 138 RFLPs, 26 SSRs, 134 AFLPs, five SSPs, and three known genes (one pyruvate decarboxylase and two lipoxygenases). The map is $3598 \mathrm{cM}$ long, with an average distance between markers of $11.8 \mathrm{cM}$, and $12.1 \%$ of the markers deviated significantly from the expected Mendelian ratio $1: 1$. The molecular markers were evenly distributed between the $\mathrm{A}$ and $\mathrm{B}$ genomes. The chromosome with the most markers is 1B (41 markers), followed by $3 \mathrm{~B}$ and $7 \mathrm{~B}$, with 25 markers each. The chro-
\end{abstract}

Communicated by P. Langridge

M.M. Nachit ( I. Elouafi · A. El Saleh · M. Labhilili A. Asbati · M. Azrak $\cdot$ H. Hazzam

ICARDA, CIMMYT/ICARDA, Durum Improvement Program, P.O. Box 5466, Aleppo, Syria e-mail: M.Nachit@CGIAR.ORG

A. Pagnotta $\cdot$ E. Iacono $\cdot$ O.A. Tanzarella $\cdot$ E. Poirceddu Dipartimento di Agrobiologia e Agrochimica,

Università della Tuscia, Via S. Camillo de Lellis, 01100 Viterbo, Italy

M. Khairallah · J.M. Ribaut

CIMMYT, Applied Biotechnology Center, Lisboa 27, A.P. 6-641, 06600 Mexico, D.F. Mexico

D. Benscher · M.E. Sorrells

Department of Plant Breeding, Cornell University, Ithaca, NY14853, USA mosomes with the fewest markers are 2A (11 markers), 5A (12 markers), and 4B (15 markers). In general, there is a good agreement between the map obtained and the Triticeae linkage consensus maps. This intraspecific map provides a useful tool for marker-assisted selection and map-based breeding for resistance to biotic and abiotic stresses and for improvement of grain quality.

Keywords Durum - RFLP - SSR - AFLP - Seed storage proteins $\cdot$ Genetic linkage mapping

\section{Introduction}

Durum wheat (Triticum turgidum L. var. durum) is an allotetraploid (genome AABB, $2 \mathrm{n}=4 \mathrm{X}=28$ ) with seven homoeologous groups. It is an especially popular cereal crop of the Mediterranean region, and its grain is used for food products such as pasta, couscous, and burghul. In contrast to both hexaploid wheat (AABBDD), for which several molecular linkage maps of chromosomes have been developed (Chao et al. 1989; Liu and Tsunewaki 1991; Devos et al. 1992, 1995; Nelson et al. 1995a,b,c; Röder et al. 1998; Van Deynze et al. 1995a; Marino et al. 1996; Jia et al. 1996) and to diploid relatives of wheat, for which linkage maps have been constructed (Gill et al. 1991; Lagudah et al. 1991; Dubcovsky et al. 1996), relatively little attention has been given to developing genetic linkage maps for durum. Indeed, it was only recently that the first linkage map of durum chromosomes, based on 65 recombinant inbred lines (RILs) and restriction fragment length polymorphism (RFLP) markers was reported (Blanco et al. 1998); microsatellite markers from hexaploid bread wheat were subsequently integrated into this genetic linkage map (Korzun et al. 1999). Further, because of low polymorphism among cultivated varieties of wheat (Anderson et al. 1993; Devos et al. 1995) most linkage maps involve interspecific crosses. However, a linkage map based on cultivated varieties may be more useful than an interspecific cross in detecting agriculturally important genes for grain yield and abiotic stress tolerance. 